\title{
Diseño de un sistema de automatización del proceso de fumigación y control de expulsión de bromuro de metilo en la agroindustria
}

\author{
David Enrique Ortega Solorzano, Juan Francisco Tisza Contreras \\ Universidad Nacional de Ingeniería, Lima, Perú.
}

Recibido el 24 de noviembre del 2019. Revisado el 7 de enero del 2020. Aceptado el 27 de enero del 2020

DOI: https://doi.org/10.33017/RevECIPeru2020.0001/

\section{Resumen}

La fumigación con bromuro de metilo es un proceso que se practica en el país debido al desarrollo de la agroindustria y el aumento de las exportaciones, pero el uso de este fumigante está restringido a nivel mundial por su daño al medio ambiente y a la salud de las personas, por ello su utilización debe seguir un estricto procedimiento. Este trabajo automatiza el proceso mejorando la calidad y rendimiento de la fumigación, además controla la expulsión del bromuro de metilo (BM) teniendo en cuenta los estándares medio ambientales y la salud de los operadores. Para ello se usa una interfaz HMI creada mediante LABVIEW que permite monitorear, registrar y controlar todo el proceso.

Descriptores: fumigación, bromuro de metilo, instrumentación, interfaz HMI, Control multi-input- multi output (MIMO)

\section{Abstract}

Fumigation with methyl bromide is a process that is practiced in the country due to the development of agribusiness and increased exports, but the use of this fumigant is restricted worldwide due to its damage to the environment and the health of people; therefore, its use must follow a strict procedure. This work automates, improves the process, the quality and the performance of the fumigation also controls the expulsion of methyl bromide taking into account the environmental means and the health of the operators. For this, an interface HMI created with LABVIEW that is used to monitor record and control the entire process.

Keywords: fumigation, methyl bromide, instrumentation, HMI interface, multi-input- multi output control (MIMO)

\section{Introducción}

El Sector Agro Exportador Peruano está pasando por una etapa de crecimiento debido a la apertura comercial del Perú. En este contexto se han desarrollado plantas de fumigación en el país que mejoran el potencial exportador al desinfectar los cultivos de las plagas y dejándolos listos para su consumo interno o para la exportación. [1]

La autoridad encargada de fiscalizar y autorizar los tratamientos de fumigación en el país es el Servicio Nacional de Sanidad Agraria - SENASA. Entre los tratamientos autorizados por SENASA figuran fumigación con bromuro de metilo, fumigación con fosfina, inmersión, aspersión - pulverización, nebulización y espolvoreo. [2]

El Bromuro de metilo es un fumigante altamente efectivo y de amplio espectro, que se convirtió en el método preferido de control para plagas y enfermedades por parte de diversos productores en todo el mundo [3]. El BM es utilizado por SENASA para el tratamiento de fumigación en cámaras a presión atmosférica normal según Anexo 6 de [2]. Este fumigante está restringido a nivel mundial debido al daño que causa a la capa de ozono y a la salud de las personas que lo puedan inhalar. Debido a ello la expulsión de este gas debe cumplir la normativa vigente [4]. 
La automatización de procesos se realiza con controladores lógicos programables - PLC's, microcontroladores - uC's o computadores mediante interfaces desarrolladas con el software comercial LabView el cual es una plataforma para diseñar sistemas de control e interfaces de usuario mediante un lenguaje de programación gráfico, lo que permite un corto tiempo de desarrollo y mantenimiento, además al estar dentro de un entorno computacional permite la aplicación de nuevos métodos de control.

\section{Proceso de fumigación}

El proceso de fumigación con bromuro de metilo consta de las siguientes etapas [2]:

1. Carga del producto

2. Etapa de dosificación

3. Etapa de fumigación

4. Etapa de aireación

5. Descarga del producto

Las etapas de Carga del producto y Descarga del producto requieren un control documentario el cual es implementado en una base de datos en Access, donde se puede tener una librería de las frutas a fumigar con la dosis recomendada, además de la relación de las pruebas realizadas.

Las etapas de dosificación, fumigación y aireación son automatizadas mediante una interfaz gráfica en el entorno LABVIEW.

\subsection{Etapa de Dosificación}

La automatización de la etapa de dosificación consta de un bucle de control (Figura 1) que calcula y controla la dosis de bromuro de metilo que ingresa a la cámara, también debe asegurar la correcta gasificación del bromuro la cual está directamente relacionada con la temperatura del vaporizador.

Para este proceso se usa la técnica de control PID, debido a que el sistema es de primer orden, con lo cual tenemos que encontrar los coeficientes $K_{c}, K_{i}$, $K_{d}$ de (1) mediante el método de ziegler nichols.

$C(s)=K_{c}+\frac{1}{K_{i} s}+K_{d} s$

\subsection{Etapa de Fumigación}

La automatización de la etapa de fumigación consta de un bucle de control (según Figura 3) que controla el tiempo de fumigación, también controla la temperatura en el interior y la distribución uniforme del bromuro (mediante una bomba que recircule el aire).

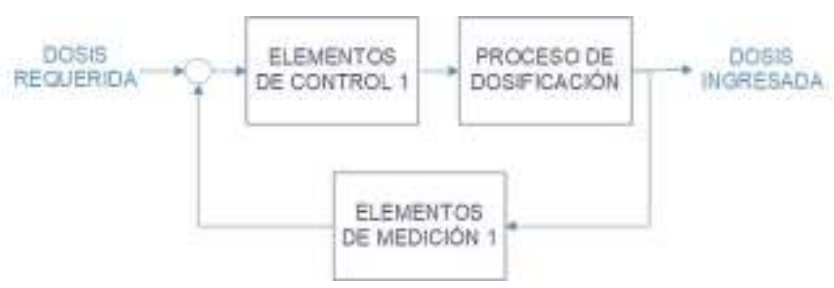

Figura 1: Diagrama de control de la etapa dosificación.

Los equipos que compondrán esta etapa están mostrados en la figura 2:

a. Tanque de Bromuro de Metilo: Dentro de este tanque se encuentra el BM a presión en estado líquido a temperatura ambiente, es importante regular la salida del tanque a una presión baja para lograr mayor exactitud en la dosificación.

b. Balanza para el Tanque de BM: Esta balanza permite obtener la cantidad de BM que está ingresando a la cámara mediante la diferencia de peso del tanque. Tiene una resolución mínima de $100 \mathrm{~g}$ [2], además de un tiempo de respuesta rápido y salida de datos vía serial.

c. Electro-válvula en la salida del Tanque de BM: Esta válvula controla la salida de BM del tanque hacia la cámara, tiene como función principal la apertura al inicio de la dosificación y su cierre cuando la balanza detecte el peso final calculado.

d. Vaporizador: Este intercambiador de calor se encarga de elevar la tempera del BM hasta su temperatura de evaporación mínima de 80 grados Celsius [2]. El serpentín en su interior debe asegurar la evaporación completa de todo el BM que ingresa en su interior. Puede ser de combustión o eléctrico, lo que convierte al vaporizador en un automatismo controlable.

e. Termómetro en el Vaporizador: Sensor que informará al controlador la temperatura del vaporizador, usualmente una termocupla tipo $\mathrm{K}$.

Es importante indicar que a la salida de esta etapa se tiene bromuro concentrado en estado gaseoso el cual estará listo para entrar a la cámara de fumigación. 
Figura 4: sistema en lazo abierto de dos entradas y dos salidas.

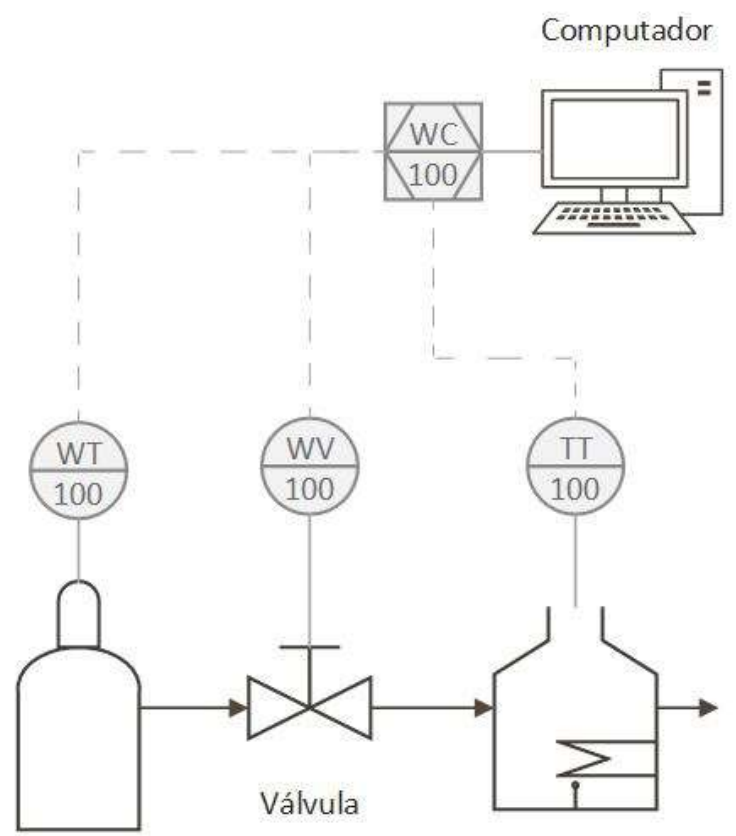

Tanque de BM

Vaporizador
Figura 2: Plano de instrumentación de la etapa dosificación.

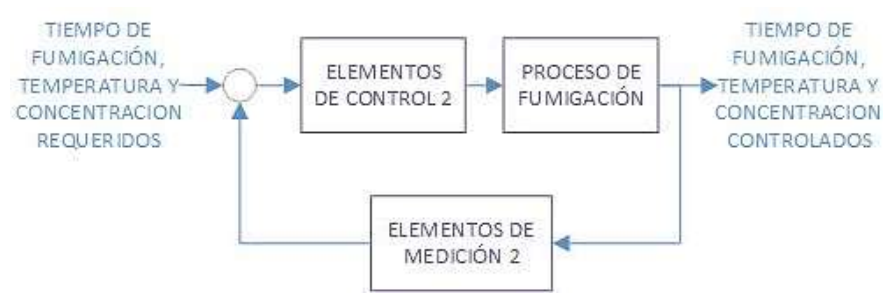

Figura 3: Diagrama de control de la etapa fumigación.

Este proceso es multi-variable lo que significa que se va a trabajar con un sistema MIMO de 2 entradas y dos salidas:

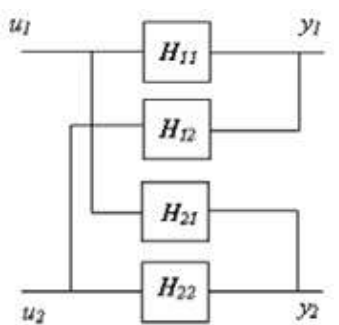

Con lo que se puede encontrar la función de transferencia:

$H(z)=\left(\begin{array}{ll}H_{11} & H_{12} \\ H_{21} & H_{22}\end{array}\right)$

Los dispositivos y equipos que componen esta etapa están mostrados en la figura 5:

a. Cámara de Fumigación: De tamaño adecuado para que el cargamento de fruta pueda ser introducido y extraído luego de su fumigación. Debe quedar completamente sellado herméticamente durante el proceso de fumigación para evitar la fuga del gas de BM.

b. Calefactor: Sube la temperatura al interior de la cámara para evitar la condensación del BM en las frutas, hasta una temperatura mínima de $19^{\circ}$ grados. Se usa un calefactor eléctrico resistivo que es controlado.

c. Refrigerador: Disminuye la temperatura al interior de la cámara para evitar la descomposición de la fruta, no permite que pase los $21^{\circ}$ grados [2]. Se usa un refrigerador eléctrico mediante bombeo de refrigerante en un radiador que es controlado.

d. Bomba de recirculación: Su función es de mantener la distribución uniforme de la concentración de BM en todo el interior de la cámara de fumigación. Se usa un motor eléctrico con paletas o una bomba centrífuga.

e. Termómetro dentro de la cámara: Sirve para obtener la temperatura en el interior de la cámara, así como el de las frutas que están siendo fumigadas. Usualmente un PT100. Con una resolución mínima de 0.1 grados Celsius.

f. Sensor de concentración de BM dentro de la cámara: Este sensor será el encargado de monitorear que se llegó a la dosis correcta y que, si el gas de BM se encuentra disperso homogéneamente en toda la cámara de fumigación, para ello deberá sensar la concentración en la puerta, medio y fondo de la cámara. 
Luego de esta etapa se tiene el gas de bromuro mezclado con aire ya usado en la fumigación.

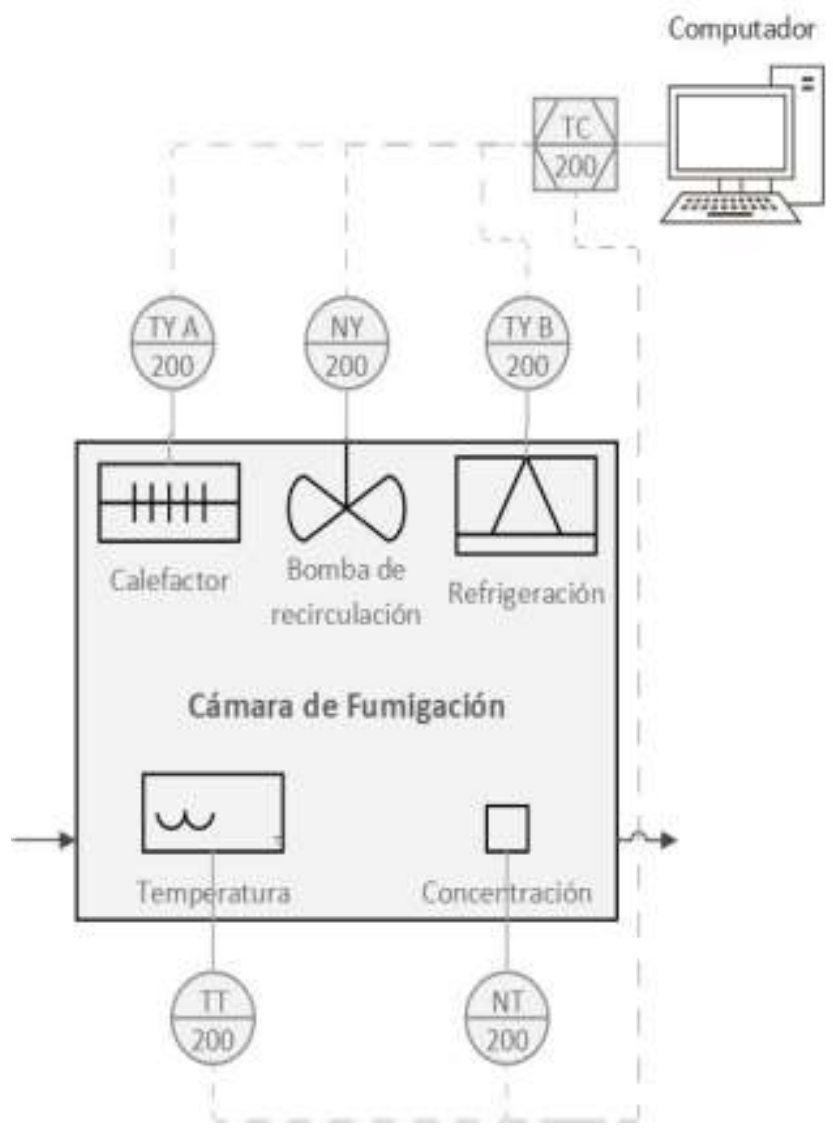

Figura 5: Plano de instrumentación de la etapa fumigación.

\subsection{Etapa de Aireación}

La automatización de la etapa de aireación consta en un bucle de control (según Figura 6) que controla la concentración en la chimenea de expulsión del gas, también controla que la concentración en el interior de la cabina sea segura antes de su apertura.

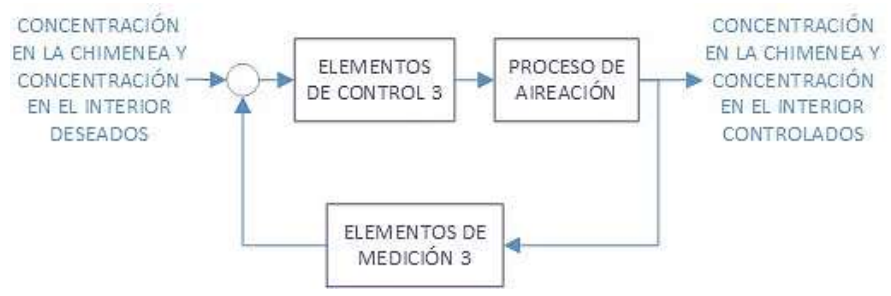

Figura 6: Diagrama de control de la etapa aireación.

Los equipos que componen esta etapa están mostrados en la figura 7: a. Servo-válvula de entrada de Aire: Cuando el aire es extraído de cámara de manera forzada mediante una bomba de extracción se generaría un vacío en el interior de la cámara, para evitar eso se tiene que tener una entrada de aire que sólo deje ingresar aire cuando haya una presión negativa en el interior, con lo cual se forma un conjunto de válvula y un check para gases.

b. Servo-válvula de salida de Aire con BM: Esta válvula sirve como controlador fundamental de la salida del aire con bromuro y es regulado automáticamente para evitar que la concentración en la chimenea pase las 500 ppm.

c. Bomba de extracción: Esta bomba es conectada inmediatamente a la salida de la cámara y sirve para la extracción directa del aire con bromuro.

d. Bomba de mezclado y expulsión: Esta bomba es la encargada de añadir aire puro al aire con bromuro para bajar su concentración y también es la que expulsa ese aire al medio ambiente.

e. Sensor de concentración de BM en la salida de la chimenea: Este sensor registra el valor de la concentración a la salida de chimenea y nos ayuda a realizar el control.

\section{Resultados}

Luego de implementar el sistema y de programar la interfaz de usuario mediante LABVIEW se obtuvo lo siguiente al terminar una prueba:

a. la dosis ingresada manualmente es similar a la registrada por los equipos durante la fumigación.

b. la temperatura de la fruta se mantuvo dentro de los valores deseados.

c. la concentración en la chimenea no supero las 500 ppm.

\section{Conclusiones}

La automatización de los procesos industriales mejora la calidad y facilitan la monitorización, el registro y el control de los valores involucrados. En este sentido la interfaz desarrollada permitiría que un solo operador controle una cámara de fumigación de más de $80 \mathrm{~m}^{3}$ desde una computadora. 
Se controló la concentración del bromuro de metilo durante su expulsión al medio ambiente lo que indica que este no tendrá un impacto negativo al medio ambiente ni a las personas 


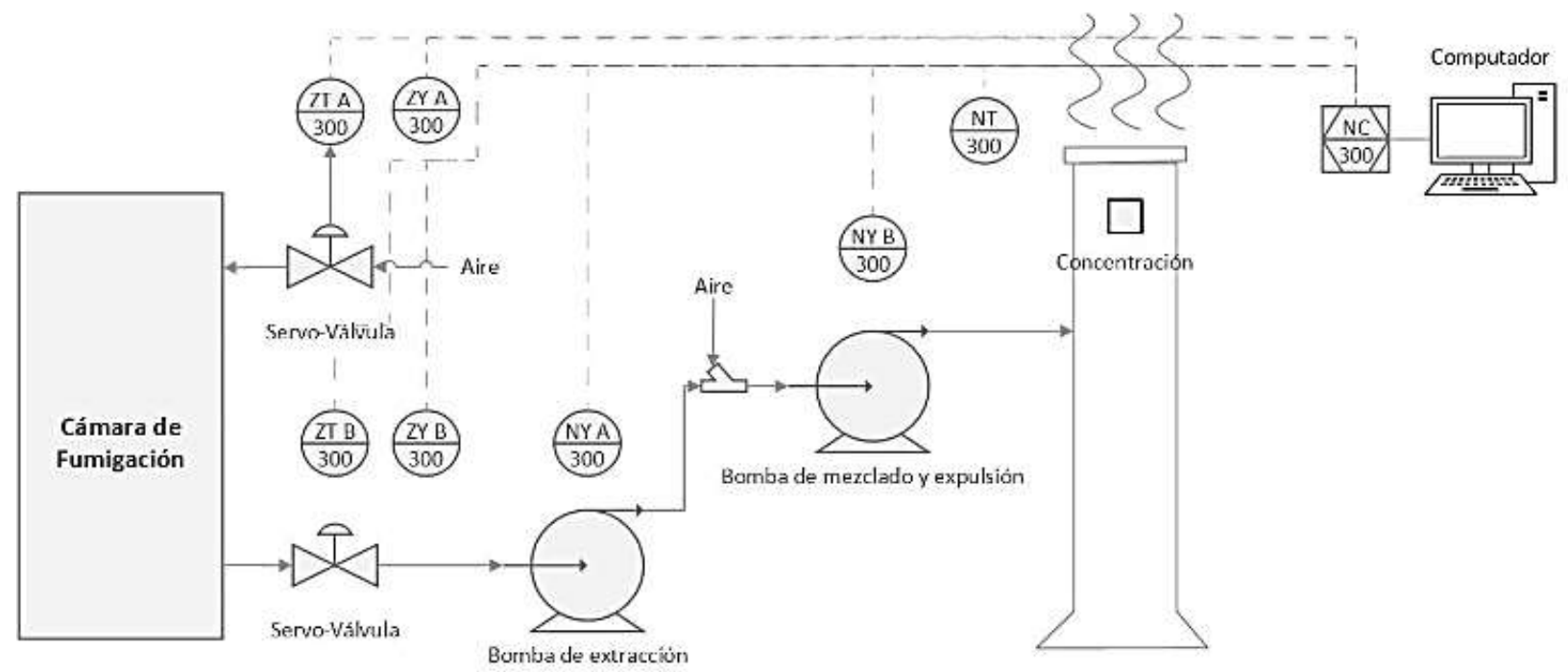

Figura 7: Plano de instrumentación de la etapa aireación.

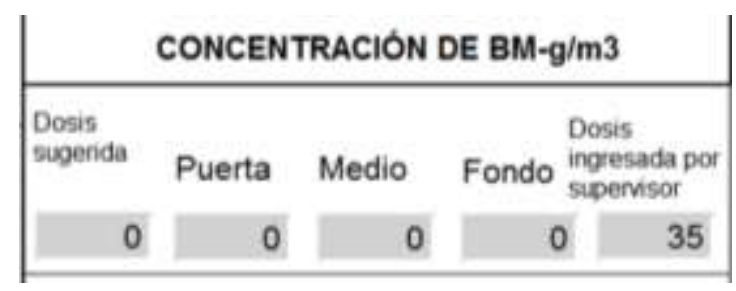

Figura 8. Dosis ingresada (a la derecha).

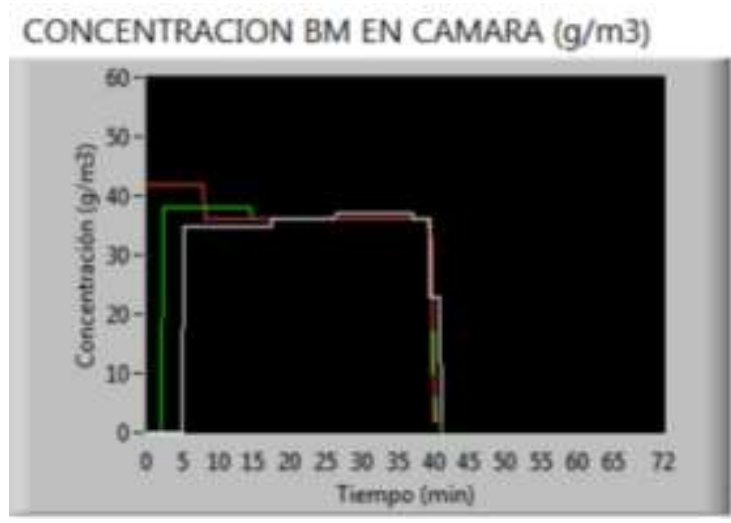

Figura 9. Dosis registrada durante el proceso.

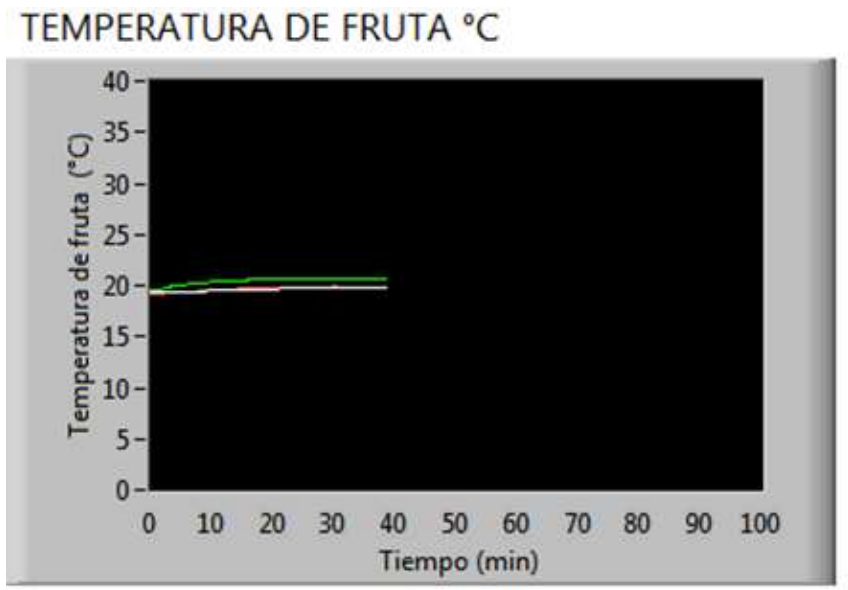

Figura 10. Temperatura de las frutas registradas.

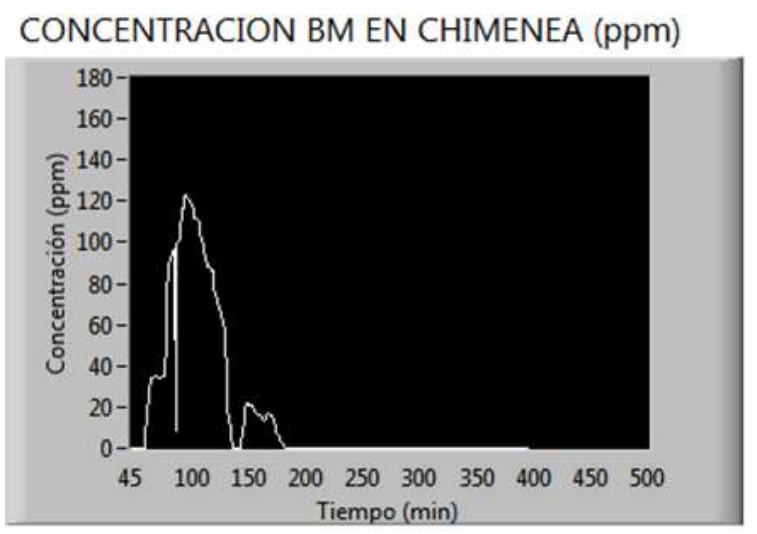

Figura 11. Concentración del bromuro de metilo registrada en la chimenea. 


\section{Referencias}

[1] R. H. Márquez, Propuesta de mejora de las medidas fito-sanitarias de cuarentena interna para alcanzar el área libre de moscas de la fruta en la región Arequipa, Tesis para optar el grado de Maestro en Ciencias Economía con mención en Gerencia Agropecuaria, Universidad Nacional de San Agustín, Arequipa, Perú, 2014.

[2] J. L. Díaz, Procedimiento: Medidas Fitosanitarias de cuarentena interna para moscas de la fruta en el Perú, Servicio Nacional de Sanidad Agraria, Perú, 2015.
[3] M. Pizano, Eliminación del Bromuro de Metilo en países en vías de desarrollo, Programa de las Naciones Unidas para el Medio Ambiente, 2014.

[4] M. Sepúlveda, L. Villanueva, Tratamiento de las emisiones de Bromuro de metilo en cámaras de fumigación. Universidad de Chile, Chile, 1996.

E-mail: david.ortega@uni.pe; jtisza@uni.edu.pe 